\title{
Building the Science of Nursing Interventions in Latin America
}

Construyendo la ciencia de las intervenciones de enfermería en Latinoamérica

Construindo a ciência das intervenções de enfermagem na América Latina

Robin Whittemore'

doi: 10.5294/aqui.2018.18.3.1

Globally, there has been a shift in health care from a focus on communicable diseases to address increased longevity of life and non-communicable diseases. Many countries worldwide not only need to prevent and control infectious diseases, but must also address the morbidity and mortality associated with chronic disease, such as diabetes and cardiovascular disease (1). Nurses play an important role in global health, both in the prevention and management of communicable and non-communicable disease. Recent initiatives in the United States advocate for nurses to practice to the full extent of their education and training within collaborative models of health care (2). This applies to nurses globally. Building the evidence-base of nursing interventions will improve nursing autonomy in practice and health outcomes. Thus, nurses need to be developing evidence to improve global health outcomes, adapting evidence-based interventions to the local context, as well as evaluating the implementation process and outcomes of evidence-based interventions.

The development and evaluation of nursing interventions requires a systematic and scientific process as many nursing interventions are complex, with multiple components, requiring specification of the content, dose, timing, and outcomes (3). Formative work is often required to better understand the needs of stakeholders (providers and patients) to optimize the protocol and ensure the feasibility and acceptability of novel interventions.

1 orcid.org/0000-0001-9434-0230. Yale School of Nursing, United States of America. robin.whittemore@yale.edu
Once the protocol has been developed, feasibility and pilot studies are necessary to determine recruitment, participation, implementation, as well as to explore the potential effect on outcomes of interest. Upon successful completion of pilot studies, intervention protocols are finalized and efficacy trials are conducted to determine the effect of the nursing intervention on mediating and proximal outcomes. While this process can take considerable time and effort, much can be learned to enhance intervention implementation, outcomes, and scalability.

Adapting evidence-based interventions from one global context to another is another important priority for nursing science and practice. Over the past 20 years, considerable evidence on the efficacy of many nursing interventions has been established. Thus, there is a need for systematic reviews of nursing interventions to be conducted to inform global health initiatives. For nurses in Latin America countries, evidence from the United States or other countries will often need to be adapted to the culture, context, and health care system. Cultural adaptation has been defined as "the systematic modification of an evidence-based treatment (EBT) or intervention protocol to consider language, culture, and context in such a way that it is compatible with the client's cultural patterns, meanings, and values" (4). Several models have been proposed to guide the cultural adaptation and evaluation of interventions that are relevant to nursing (5). Steps to this process include information gathering, preliminary adaptation design of intervention, preliminary adaptation test of inter- 
vention (for feasibility and acceptability), adaptation refinement, and lastly implementation and evaluation.

Latin American nursing research has grown significantly in the past several decades (6). Strategies to continue to enhance the development and implementation of culturallyrelevant evidence-based nursing interventions include: building the research infrastructure of nursing research through participation in conferences and workshops such as the Pan American Nursing Research Colloquium ${ }^{2}$ and/or international research meetings (nursing and interdisciplinary); building collaborations across universities and between universities and clinical settings; and disseminating research results through conference presentations and publications. There is much to be gained by the development and evaluation of nursing interventions world-wide.

\section{Referencias}

1. World Health Organization. Chronic diseases and health promotion: Integrated chronic disease prevention and control. 2018. Available from: http://www.who.int/chp/about/integrated_cd/en/

2. Institute of Medicine. Assessing progress in the Institute of Medicine report: The future of nursing. Washington D.C.: National Academy of Sciences; 2015.

3. Whittemore R, Grey M. The systematic development of nursing interventions. Journal of Nursing Scholarship. 2002; 34: $115-120$.

4. Bernal G, Jimenez-Chafey MI, Domenech-Rodríguez MM. Cultural adaptation of treatments: A resource for considering culture in evidence-based practice. Professional Psychology: Research and Practice. 2009; 40: 361-368.

5. Barrera M, Castro FG, Strycker LA, Toobert D. Cultural adaptations of behavioral health interventions: A progress report. Journal of Consulting and Clinical Psychology. 2013; 81: 196-205.

6. Mendoza-Parra S, Paravic-Klijn T, Muñoz-Muñoz AM, Barriga OA, Jiménez-Contreras E. Visibility of Latin American nursing research (1959-2005). Journal of Nursing Scholarship. 2009; 41: 54-63.

2 More information in: https://www.caipe.org/news/xvi pan american nursing research colloquium 